\title{
Type specimens of true bugs (Hemiptera: Heteroptera) housed in the Museu de Zoologia da Universidade de São Paulo, Brazil
}

\author{
Renan Carrenho ${ }^{1,4}$; Higor D.D. Rodrigues ${ }^{1,2,5}$; Adriana Carneiro de Lima ${ }^{3,6}$ \& Cristiano F. Schwertner ${ }^{1,3,7}$
}

\author{
1 Universidade de São Paulo (USP), Museu de Zoologia (MZUSP). São Paulo, SP, Brasil. \\ 2 Instituto Oswaldo Cruz (IOC), Laboratório de Biodiversidade Entomológica. Rio de Janeiro, RJ, Brasil. \\ ${ }^{3}$ Universidade Federal de São Paulo (UNIFESP), Departamento de Ecologia e Biologia Evolutiva. Diadema, SP, Brasil. \\ ${ }^{4}$ ORCID: http://orcid.org/0000-0003-4268-7309. E-mail: renan.carrenho@gmail.com (corresponding author) \\ ${ }^{5}$ ORCID: http://orcid.org/0000-0002-9649-4142. E-mail: higorddr@gmail.com \\ ${ }^{6}$ ORCID: http://orcid.org/0000-0001-8390-9313. E-mail: drilima1001@gmail.com \\ 7 ORCID: http://orcid.org/0000-0001-5104-4925. E-mail: schwertner@unifesp.br
}

\begin{abstract}
This paper provides a record of the type specimens of Heteroptera (Insecta: Hemiptera) housed in the Museu de Zoologia da Universidade de São Paulo, Brazil (MZUSP), with information on 65 primary types and 316 secondary types. These type specimens represent 116 nominal species, currently classified in five infraorders and 14 families. The taxa are presented alphabetically, followed by bibliographic citation, type status, preservation method, collection data, and taxonomic remarks when appropriate.
\end{abstract}

Key-Words. Heteroptera; True bugs; Taxonomy; List of type material; MZUSP.

\section{INTRODUCTION}

The insects commonly known as true bugs (Heteroptera) currently comprise seven monophyletic lineages with infraorder status, which account for more than 40,000 described species (Weirauch \& Schuh, 2011). Members of the infraorders Enicocephalomorpha and Dipsocoromorpha are usually found on the surface or underneath soil debris and leaf litter; members of Gerromorpha, Nepomorpha and Leptopodomorpha are associated with aquatic environments; and members of Cimicomorpha and Pentatomomorpha, known as terrestrial true bugs, live in a wide variety of habitats (Schuh \& Slater, 1995).

The Museu de Zoologia da Universidade de São Paulo (MZUSP) is a major biodiversity repository in Latin America, and houses a wealth of research specimens, providing thousands of specimens to worldwide specialists each year (Landim, 2011). MZUSP holdings account for over 10 million animal specimens, with approximately seven thousand primary types (Ulysséa et al., 2015). A series of catalogues have been recently published to document this material (e.g., Valim, 2009; Onody et al., 2014; Falaschi et al., 2016, 2018; Santos et al., 2017). The entomology collection at MZUSP houses approximately 2.2 million mounted specimens with an additional 2.5 million that await to be processed (Taddei et al., 1999; Ferreira et al., 2016).

Historically, Hemiptera is one of the few collections at the MZUSP that never employed a full-time specialist curator. Although renowned Hemiptera researchers worked in the city of São Paulo in the first half of the $20^{\text {th }}$ century (e.g., Adolf Hempel, José Pinto da Fonseca, Edson J. Hambleton, and Oscar Monte), all of them were formally affiliated with the Instituto Biológico (IB), a research division of the Department of Agriculture of the State of São Paulo, which was later incorporated into the Secretariat of Agriculture and Supplies. Like many other insect groups, the earliest Heteroptera specimens from MZUSP date back to the early $20^{\text {th }}$ century and were mainly collected by Hermann von Ihering, Hermann von Lüderwaldt, Adolf Hempel and Ernest Garbe (C. Campaner, personal communication). At that time, the entomology collection was part of the Museu Paulista (Landim, 2011), which housed both historical documents and natural history specimens. However, it was later 
incorporated into a newly-established zoology museum (MZSUP) in the late 1960s (Evangelista et al., 2014). This holding includes an extensive amount of species described and/or identified by specialists with great historical significance to Hemiptera systematics (e.g., Albino M. Sakakibara; Olivia Evangelista; Rodney R. Cavichioli; José Cândido de M. Carvalho; José De Carlo; Felipe F.F. Moreira; Nico Nieser; Carl Drake; Harry Brailovsky; Nicholas Kormilev; Jocélia Grazia).

The first catalogue of hemipteran types at the MZUSP was recently published for treehoppers (Auchenorrhyncha: Membracidae) (Evangelista et al., 2014). Following a recommendation by the International Code of Zoological Nomenclature (72F.4), we hereby provide a list of the Heteroptera types as a contribution to the knowledge of the institution's holdings.

\section{MATERIAL AND METHODS}

This catalogue contains information on all primary and secondary types of Heteroptera held by the Museu de Zoologia da Universidade de São Paulo. Infraorders, families, genera, and species are presented in alphabetical order. Specimen data was extracted from labels, and when necessary, annotated based on original descriptions. The Heteroptera types of this collection do not have unique accession numbers.

The data provided for the type specimens are organized according to the following sequence: genus, species epithet, author, year of publication, page and figure(s) in the original publication, type status, gender, wing traits (if polymorphic), method of preservation, locality data and collector. Types are listed according to their original combination and, when applicable, subsequent taxonomic changes or type designations are indicated. Label transcriptions are cited verbatim, and when abbreviated or incomplete, annotations are provided within brackets to prevent any ambiguity. In some cases, type specimens were originally designated to other institutions, but due to various historical factors, were later deposited at MZSUP. Therefore, this catalogue lists all type specimens whose current repository is the MZUSP, even when it has been stated otherwise in the original description.

\section{RESULTS}

A total of 65 primary types ( 61 holotypes and four lectotypes) and 316 secondary types (307 paratypes, 6 allotypes and 3 paralectotypes) were found (Table 1). These type specimens represent 116 nominal species across 5 infraorders and 14 heteropteran families: Cimicomorpha (Miridae, Reduviidae and Tingidae), Enicocephalomorpha (Enicocephalidae), Gerromorpha (Gerridae and Veliidae), Nepomorpha (Potamocoridae, Naucoridae, Nepidae and Notonectidae), and Pentatomomorpha (Aradidae, Coreidae, Pentatomidae and Rhyparochromidae). Type specimens are listed below.
Table 1. Type material of Heteroptera housed in the Museu de Zoologia da Universidade de São Paulo.

\begin{tabular}{|c|c|c|c|c|c|c|}
\hline Infraorder & Family & $\begin{array}{l}\text { 홓 } \\
\text { 흥 } \\
\text { 오 }\end{array}$ & 冚 & 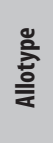 & 总 & 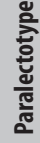 \\
\hline \multirow[t]{3}{*}{ Cimicomorpha } & Miridae & 13 & - & - & 25 & - \\
\hline & Reduviidae & 8 & - & - & 23 & - \\
\hline & Tingidae & - & - & - & 11 & - \\
\hline Enicocephalomorpha & Enicocephalidae & 1 & - & - & 3 & 一 \\
\hline \multirow[t]{2}{*}{ Gerromorpha } & Gerridae & 1 & - & 1 & - & - \\
\hline & Veliidae & 12 & 4 & 1 & 121 & 3 \\
\hline \multirow[t]{4}{*}{ Nepomorpha } & Naucoridae & 2 & - & - & 11 & - \\
\hline & Nepidae & 3 & - & 3 & 1 & 一 \\
\hline & Notonectidae & 1 & - & 1 & 2 & - \\
\hline & Potamocoridae & - & - & - & 10 & 一 \\
\hline \multirow[t]{4}{*}{ Pentatomomorpha } & Aradidae & 6 & - & - & 2 & - \\
\hline & Coreidae & 1 & - & - & 12 & - \\
\hline & Pentatomidae & 12 & - & - & 84 & - \\
\hline & Rhyparochromidae & 1 & - & 一 & 2 & 一 \\
\hline Total & & 61 & 4 & 6 & 307 & 3 \\
\hline
\end{tabular}

\section{Order Hemiptera Linnaeus, 1758 \\ Suborder Heteroptera Latreille, 1810 \\ Infraorder Cimicomorpha Leston et al., 1954 \\ Miridae Hahn, 1831 \\ Adparaproba yungensis Carvalho, 1990}

Adparaproba yungensis Carvalho, 1990: 445-447, figs. 1-4.

Holotype: $\sigma^{7}$, glued to pinned paper; Bolivia, Puenta Villa, Yungas 1200 m[e]t[e]r[s]. XII.1955, Dirings col.

Paratypes: 10", glued to pinned paper; Bolivia, Chulumani Yungas 1700 m[e]t[e]r[s]. XII.1956, Dirings col. 19, glued to pinned paper; Bolivia, El Palomar Chaparé $900 \mathrm{~m}[\mathrm{e}] \mathrm{t}[\mathrm{e}] \mathrm{r}[\mathrm{s}]$. IX.1956, Dirings col. 20', glued to pinned paper; Bolivia, La Paz, Coroico Yungas 1700 m[e]t[e]r[s]. XII.1955, Dirings col.

\section{Collaria boliviana Carvalho, 1990}

Collaria boliviana Carvalho, 1990: 447-449, figs. 5-8.

Holotype: \&, glued to pinned paper; Bolivia, Rurrenabaque, Beni, 175 m[e]t[e]r[s]. X.1956, Dirings col.

Paratypes: $3 \sigma^{x}$, glued to pinned paper; same data as holotype.

Remarks: The original description states that all the specimens were collected in [19]61, but all labels read 1956.

Clypeocoris amazonicus Carvalho, 1989

Clypeocoris amazonicus Carvalho, 1989: 444, figs. 1-5. 
Holotype: $\sigma^{\prime \prime}$, glued to pinned paper; [Brazil], AM[azonas], Maturaca, alto Rio Cauaburi 28.XII.[19]62-05.I.[19]63, J. Bechyné col.

\section{Cylapocoroides centralis Carvalho, 1989}

Cylapocoroides centralis, Carvalho, 1989: 446, figs. 6-9.

Paratype: $10^{\prime}$, glued to pinned paper; Brasil, Rio de Janeiro, Rio Grande, Repr[esa]. No date, F.M. Oliveira col.

\section{Cyrtocapsus bolivianus Carvalho, 1990}

Cyrtocapsus bolivianus, Carvalho, 1990: 450, fig. 9.

Holotype: 9 , glued to pinned paper; Bolivia, Puente Villa Yungas $1200 \mathrm{~m}[\mathrm{e}] \mathrm{t}[\mathrm{e}] \mathrm{r}[\mathrm{s}]$. XII.1955, Dirings col.

Paratypes: 3\%; same data as holotype.

\section{Cyrtotylus catarinenses Carvalho, 1989}

Cyrtotylus catarinenses Carvalho, 1989:448-450, figs. 12-16.

Holotype: $\sigma^{\pi}$, glued to pinned paper; Brasilien [Brazil], Nova Teutonia, $27^{\circ} 11^{\prime} \mathrm{O}[\mathrm{S}], 52^{\circ} 23^{\prime} \mathrm{L}$ [W], 300-500 m. XII.1949, Fritz Plaumann col.

Remarks: According to the original description, the holotype was collected in Alchornea sidifolia (Euphorbiaceae).

\section{Herdonoides paulistanus Carvalho, 1989}

Herdonoides paulistanus Carvalho, 1989: 451, figs. 17-22.

Holotype: $\sigma^{n}$, pinned; [Brazil], São Paulo, Campos do Jordão. 23.XII.1944, F. Lane col.

\section{Horciasoides ouropretanus Carvalho, 1989}

Horciasoides ouropretanus Carvalho, 1989: 451-453, figs. 23-26.

Holotype: $\sigma^{\prime}$, glued to pinned paper; [Brazil], MG [Minas Gerais], Topázios Ouro Preto, 22.II.1962, J. Bechyné col.

Paratype: $10^{\pi}$, same data as holotype.

\section{Knightonia incachaca Carvalho, 1990}

Knightonia incachaca Carvalho, 1990:450-452, figs. 10-15.

Holotype: + , glued to pinned paper; Bolivia, Incachaca, Cochabamba, 2100 m[e]t[e]r[s]. VIII.1956, Dirings col.
Remarks: The original description states that the holotype was collected at 3100 meters.

\section{Liliancoris nigrus Carvalho, 1989}

Liliancoris nigrus Carvalho, 1989: 454-455, figs. 27-31.

Holotype: $\$$, glued to pinned paper; Brasil, MG [Minas Gerais], Mar de Espanha, 27-28.II.1962, J. Bechyné col.

Paratypes: 19 , glued to pinned paper; same data as holotype. 1\%, glued to pinned paper; Brasil, Barueri, São Paulo. 13.I.1962, K. Lenko col.

Remarks: In the original description, all the paratypes are listed as having the same collection data as the holotype.

\section{Lygus omnivagus Knight, 1917}

Lygus omnivagus Knight, 1917: 627-629, fig. 201.

Paratypes: 10, glued to pinned paper; [United States of America], N.Y. [New York], Conesus Lake, June [VI] 23.1916, H.H Knight col. 1\%, glued to pinned paper; [United States of America], N[ew].Y[ork], Conesus Lake, June [VI] 23.1916, H.H Knight col.

\section{Lygus tiliae Knight, 1917}

Lygus tiliae Knight, 1917: 613-615, fig. 193.

Paratypes: 2\%, glued to pinned paper; [United States of America], N.Y. [New York], Portage, June, 27, 1915, H.H Knight col.

\section{Lygus vanduzeei Knight, 1917}

Lygus vanduzeei Knight, 1917: 565-567, fig. 159.

Paratypes: $10^{\pi}$, pinned; [United Stated of America], Rock City, N.Y. [New York], Cattaraugus Co[unty], July, 05, 1915 , H.H Knight col. 19 pinned; [United Stated of America], Rock City, N[ew].Y[ork], Cattaraugus Co[unty], July, 04, 1915, H.H Knight col.

\section{Poeas goiana Carvalho, 1989}

Poeas goiana Carvalho, 1989: 455, figs. 35-38.

Holotype: $\sigma^{\pi}$, glued to pinned paper; Brasil, Goias, Faz[enda]. Cachoeirinha Jataí, X.1962, Exp[edição]. Dep[artamento]. Zool[ogia].

Paratype: 1\%, pinned; Brasil, Goias, Faz[enda]. Aceiro Jataí, X.1962, Exp[edição]. Dep[artamento]. Zool[ogia]. 
Remarks: In the original description, all paratypes are said to be collected in 1862, however, these specimens were collected by staff from the Department of Zoology of Museu Paulista in 1962.

\section{Prepops rurrenabaquensis Carvalho \& Costa, 1991}

Prepops rurrenabaquensis Carvalho \& Costa, 1991:56, fig. 14.

Holotype: $\$$, glued to pinned paper; Bolivia, Beni, Rurrenabaque, 175 m[e]t[e]r[s], X.1956, Dirings col.

Remarks: The original description specifies that the holotype was collected in [1]961, 178 meters, however, the label clearly states 1956 and 175 meters respectively.

\section{Prepops ubirajarai Carvalho, 1989}

Prepops ubirajarai Carvalho, 1989: 455-457, figs. 39-42.

Paratype: 10", pinned; [Brazil], Faz[enda]. Iracema, Ribeirão Preto, 11.XII.1953, Barretto col.

Remarks: The original description states that the holotype was collected in 11.XII.1956 and deposited in the author's private collection.

\section{Proba saltensis Carvalho \& Costa, 1989}

Proba saltensis Carvalho \& Costa, 1989: 741, figs. 20-23.

Paratype: 19, glued to pinned paper; Bolivia, Chulumani Yungas, XII.1955, Dirings col.

\section{Saileria sulina Carvalho, 1989}

Saileria sulina Carvalho, 1989: 457-459, figs. 43-46.

Holotype: $\sigma^{\pi}$, glued to pinned paper; Brasilien [Brazil], Nova Teutonia, $27^{\circ} 11^{\prime} \mathrm{S}, 52^{\circ} 23^{\prime} \mathrm{W}$, 300-500 m. XII.1949, Fritz Plaumann col.

Paratype: 19 , glued to pinned paper; same data as holotype.

Remarks: According to the original publication, the holotype was collected in Alchornea sidifolia (Euphorbiaceae).

\section{Vitoriacoris nigrus Carvalho, 1989}

Vitoriacoris nigrus Carvalho, 1989: 459-460, figs. 48-51.

Holotype: 19, glued to pinned paper; Brasil, S[ão]. Paulo, Ilha da Vitória, 16-27.III.1964, Exp[edição]. Dep[artamento]. Zool[ogia].
Paratype: 19 , glued to pinned paper; same data as holotype.

\section{Reduviidae Latreille, 1807}

Agricocleptes wygodzinskyi Prosen \& Martínez, 1953

Agricocleptes wygodzinskyi Prosen \& Martínez, 1953: 53-56, fig. 1.

Paratypes: 19, glued to pinned paper; Bolivia, Cochabamba, Alr[ededores]. de la ciudad, no date Martinez and Prosen col. Bolivia Dep. 20', 49, pinned; Bolivia, Dep[artamento de]. Cochabamba 2600 m Zischka, III.1950, Dirings col.

Remarks: No types specimens are designated to MZUSP according to the original description.

\section{Apiomerus costalimai Prosen \& Martínez, 1955}

Apiomerus costalimai Prosen \& Martínez, 1955: 43-46, figs. 1-3.

Paratype: 19 , Argentina, Buenos Aires, Tandil, $37.3167^{\circ} \mathrm{S}$, 59.1500 W, 01 Jan 1952, no collector data.

\section{Brontostoma diringshofeni Gil-Santana \& Baena, 2009}

Brontostoma diringshofeni Gil-Santana \& Baena, 2009: 46-48, figs. 28-46.

Holotype: $\sigma^{\prime}$, pinned, with male genitalia in a vial, pinned separately; Bolivia, Santa Cruz, 500 m, x-1955, [ex-coll.] Dirings[hofen], Zischka, col.

\section{Dohrnemesa lanei Wygodzinsky, 1945}

Dohrnemesa lanei Wygodzinsky, 1945: 252-255, figs. 19, 21-33.

Holotype: $\sigma^{x}$, pinned; [Brazil], São Paulo, Campos de Jordão, 26.XII.1944, F. Lane col.

\section{Melanolestes lugens Coscarón \& Carpinteiro, 1993}

Melanolestes lugens Coscarón \& Carpinteiro, 1993: 369, figs. 25-38.

Paratypes: 19, pinned; [Brazil], MT [Mato Grosso do Sul], Três Lagoas, Faz[enda]. Canaã, Marg[em]. esq[uerda]. rio Sucuriu, I.1967 F. Lane col. 19, Pinned; [Brazil], MT [Mato Grosso], Utiariti 325 m, rio Papagaio, VII-VIII. [1]961, K. Lenko col. 19, pinned; Brasil, MT [Mato Grosso], Barra do Tapirapé, 21-31.XII.1965, B. Malkin col. 


\section{Notocyrtus amapaensis Carvalho \& Costa, 1992}

Notocyrtus amapaensis Carvalho \& Costa, 1992: 73-74, figs. $2,7,11$.

Holotype: ơ, pinned; Brasil, Amapá, Serra do Navio, IX.1957, K. Lenko col.

\section{Notocyrtus costai Gil-Santana \& Forero, 2009}

Notocyrtus costai Gil-Santana \& Forero, 2009: 62-63, figs. 7-13.

Holotype: $\$$, pinned, with right middle leg glued to a paper board, pinned with the specimen; Surinam, Marowijne dist.[rict], Langaman Kondre, VIII-1965, B. Malkin col.

\section{Notocyrtus dispersus Carvalho \& Costa, 1992}

Notocyrtus dispersus Carvalho \& Costa, 1992: 75-76, figs. 4, 8, 13.

Holotype: $\sigma^{x}$, pinned; [Brazil], S[ão]. Paulo, [Municipality of Bebedouro], Andes, II.[19]55, M. Carrera col.

Paratypes: $20^{\prime}, 19$ pinned; same data as holotype. $10^{\prime \prime}$, pinned; [Brazil], Goias, Jataí, I.[19]55, M. Carrera col. 10", pinned; [Brazil] Alagoas, Mangabeira, XI.1952, CamargoAndr. col. 19, pinned; Brasil, Minas Gerais, Sete Lagoas, III.1963, F. Werner, U. Martins, L. Silva col. 10', pinned; Brasil, MG [Minas Gerais], Serra [do] Caraça, XI.[1]961, 1880 m, Kloss, Lenko, Martins \& Silva col.

\section{Notocyrtus paraenses Carvalho \& Costa, 1992}

Notocyrtus paraenses Carvalho \& Costa, 1992: 78, figs. 6 , 15.

Holotype: 19, glued to pinned plastic; Brasil, Pará, Ananindeua, I.1963, Machado \& Pereira col.

\section{Pessoaia piratoides Costa Lima, 1940}

Pessoaia piratoides Costa Lima, 1940: 489-490.

Holotype: $\$$, pinned with genitalia and leg, slide mounted; [Brazil], Leopoldo Bulhões, no date, Prof. Pessôa col.

\section{Pseudopothea paulai Gil-Santana, 2015}

Pseudopothea paulai Gil-Santana, 2015: 543-551, figs. 3-30.
Paratype: $1 \%$, pinned, with antennae glued to pinned paper; Brasil, CE [Ceará], Carquejo, III.1963, Dirings[hofen] col.

\section{Rasahus brasiliensis Coscarón, 1983}

Rasahus brasiliensis Coscarón, 1983: 108-109, fig. 17, A-H.

Paratypes: $1 \sigma^{\pi}$, pinned; Brasil, Pará, Ponte Grossa, XII. [19]38 Camargo col. 10", pinned; BR[azil], RJ [Rio de Janeiro]., Angra dos Reis, Faz[enda]. Japuhyba VII-VIII. 1945, L.T.F. col. 19, pinned; Brasil, S.P. [São Paulo], Ilha dos Buzios, 16.X-04.XI [1]963 Exp[edição]. Dep[artamento]. Zool[ogia].

\section{Tydides imitator Lent, 1955}

Tydides imitator Lent, 1955: 168-172, figs. 8-11, 13, 16, 19 , $22,24$.

Paratype: 10', pinned; [Brazil], [Rio Grande do Sul], Porto Alegre, no date and collector data.

\section{Tingidae Laporte, 1832} Corythucha pellucida Drake \& Hambleton, 1938

Corythucha pellucida Drake \& Hambleton, 1938: 67-68.

Paratype: 10', glued to pinned paper; Brasil, São Paulo, Campinas, 10.III.1936, E.J. Hambleton col.

\section{Gargaphia paraguayensis Drake \& Poor, 1940}

Gargaphia paraguayensis Drake \& Poor, 1940: 229-230.

Paratypes: 20', glued to pinned paper; Paraguay, Horqueta, 1938, Alberto Schulze col.

Remarks: Type specimens were said to belong to the C.J. Drake 1956 collection.

\section{Leptobyrsa bruchi Drake, 1928}

Leptobyrsa bruchi Drake, 1928: 73-74.

Paratype: 1\%, glued to pinned paper; [Argentina], Cordoba 12-13-21, no date and collector data.

Remarks: Type specimens were said to belong to the C.J. Drake 1956 collection.

\section{Leptobyrsa tersa Drake \& Hambleton, 1935}

Leptobyrsa tersa Drake \& Hambleton, 1935: 148. 
Paratype: 19, glued to pinned paper; Brasil, São Paulo, São Paulo, 26.8[VIII].1934, E.J. Hambleton col.

\section{Leptopharsa machalana Drake \& Hambleton, 1946}

Leptopharsa machalana Drake \& Hambleton, 1946: 12-13.

Paratype: 10', glued to pinned paper; Ecu[ador], Machala, Sept[ember] [IX]. 27, 1944, E.J. Hambleton col.

Remarks: Type specimens were said to belong to the C.J. Drake 1956 collection.

\section{Teleonemia molinai Drake, 1940}

Teleonemia molinai Drake, 1940: 243.

Paratype: 10", glued to pinned paper; Paraguay, Horqueta 1938, Alberto Schulze col.

Remarks: Type specimens were said to belong to the C.J. Drake 1956 collection.

\section{Tigava bombacis Drake \& Poor, 1938}

Tigava bombacis Drake \& Poor, 1938: 32-33.

Paratypes: 2q, 10", glued to pinned paper; Brazil, Minas Geraes [Gerais], Viçosa, 06.V.[19]34, E.J. Hambleton col.

Remarks: Type specimens were said to belong to the C.J. Drake 1956 collection.

\section{Vatiga cassiae Drake \& Hambleton, 1934}

Vatiga cassiae Drake \& Hambleton, 1934: 440-441.

Paratype: $10^{\circ}$, glued to pinned paper; Brasil, Minas Geraes [Gerais], Viçosa 28.IV.[19]33, E.J. Hambleton col.

Remarks: Type specimens were said to belong to the C.J. Drake 1956 collection.

\section{Infraorder Enicocephalomorpha Stichel, 1955 Enicocephalidae Stål, 1860}

Enicocephalus lenkoi Wygodzinsky \& Schmidt, 1991

Enicocephalus lenkoi Wygodzinsky \& Schmidt, 1991: 119-121, figs. 71, 82, 83.

Holotype: $\sigma^{n}$, glued to pinned paper; Brasil, São Paulo, Barueri. 19.III.1967, K. Lenko col.

Paratypes: 19, 2 nymphs; glued to pinned paper; same data as holotype.

\section{Infraorder Gerromorpha Popov, 1971 \\ Gerridae Leach, 1815 \\ Neogerris kontos Nieser, 1994}

Neogerris kontos Nieser, 1994: 31-34, figs. 5, 7-9, 15.

Holotype: Apterous o', pinned; Brasil, M.G. [Minas Gerais], Gouveia, 20.III.[19]94, leg Nieser, N9425, 1300 m asl., pond.

Allotype: Apterous $\uparrow$, pinned; same data as holotype.

Remarks: In the original description, the author included additional information about the type-locality: "pond alongside road (apparently semi-artificial by the road blocking some water flow) rich emergente and aquatic vegetation, $\mathrm{pH} 5.5$, conductivity $10 \mu \mathrm{S}, \mathrm{N} .9495^{\prime \prime}$.

\section{Veliidae Brullé, 1836 \\ Euvelia mazzucconiae Aristizábal-García et al., 2015 \\ Euvelia mazzucconiae Aristizábal-García, Floriano, Moreira \& Bispo, 2015: 592-594, figs. 1-6.}

Paratypes: $10^{n}, 1 \%$, glued to pinned paper; Colombia, Antioquia, Mpio, Necoclí, Mar Caribe en el Golfo de Urubá, 07.IX.[19]95, Aristizábal-G col.

Remarks: In the original description, all paratypes are deposited in the Instituto de Ciencias Naturales de La Universidad Nacional de Colombia, Bogota, and in the eQual Collection, Bogota, both in Colombia.

\section{Oiovelia chenae Rodrigues \& Melo, 2014}

Oiovelia chenae Rodrigues \& Melo in Rodrigues et al., 2014a: 84-87, figs. 47-49, 59-61, 74, 78.

Paratypes: 3 macropterous $\sigma^{\star}, 3$ macropterous $\$$, glued to pinned paper; Brasil, Pará, Alter do Chão, $10 \mathrm{~km}$ Santarém, Igarapé Jutuaranã, on foam masses, $30 \mathrm{~m}$, $02^{\circ} 32^{\prime} 04.5^{\prime \prime} \mathrm{S}, 54^{\circ} 54^{\prime} 33.0^{\prime \prime} \mathrm{W}, 01 . \mathrm{X} .2012$, A. Fernandes col.

\section{Oiovelia hamadae Rodrigues \& Melo, 2014}

Oiovelia hamadae Rodrigues \& Melo in Rodrigues et al., 2014a: 87-89, figs. 50-51, 62-64, 75, 79.

Paratypes: 3 macropterous ơ, 3 macropterous $\$$, glued to pinned paper; Brasil, Amazonas, Reserva Florestal Adolpho Ducke, Igarapé Acará, on foam masses, 22.VI.2011, H.D.D. Rodrigues col.

\section{Oiovelia machadoi Rodrigues \& Moreira, 2016}

Oiovelia machadoi Rodrigues \& Moreira, 2016a: 154-156, figs. 1-8, 10. 
Holotype: Macropterous $\sigma^{n}$, glued to pinned paper; Brasil, São Paulo, Parque Estadual da Serra do Mar, Cachoeira da Pedra Furada, on foam masses, $23^{\circ} 42^{\prime} 16.4^{\prime \prime} \mathrm{S}$, 4602'42.8"W, 725 m, 23.VIII.2014, H. Rodrigues col.

Paratypes: 5 macropterous $\sigma^{\prime \prime}, 5$ macropterous $q$, glued to pinned paper; same data as holotype.

\section{Oiovelia pydanieli Rodrigues \& Melo, 2014}

Oiovelia pydanieli Rodrigues \& Melo, in Rodrigues et al., 2014a: 89-91, figs. 52-54, 65-67, 76, 80.

Paratypes: 3 macropterous $\sigma^{n}, 3$ macropterous $q$, glued to pinned paper; Brasil, Roraima, Área Indígena Yanomami, Serra de Surucucu, $02^{\circ} 49^{\prime} 53^{\prime \prime} \mathrm{N}, 63^{\circ} 38^{\prime} 19^{\prime \prime} \mathrm{W}$, 26.XI.1991, V. Py-Daniel \& U. Barbosa col.

\section{Oiovelia spumicola Spangler, 1986}

Oiovelia spumicola Spangler, 1986: 438-443, 446, figs. 1-12, 21.

Paratype: 1 apterous $\&$, glued to pinned paper; Venezuela, T.F. Amaz.[onas], Cerro de la Neblina, In masses of foam in mountain stream, Camp XI, 1450 m., $00^{\circ} 52^{\prime} \mathrm{N}$, $65^{\circ} 58^{\prime}$ W, 25-28 February 1985, P.J. \& P.M. Spangler \& R.A. Faitoute col.

Remarks: In the original description, the author does not include the MZUSP collection as repository of any specimen of the type series.

\section{Oiovelia viannai Rodrigues \& Melo, 2014}

Oiovelia viannai Rodrigues \& Melo in Rodrigues et al., 2014a: 91-93, 95-97, figs. 55-58, 68-70, 77.

Holotype: Apterous $\sigma^{\prime \prime}$, glued to pinned paper; Brasil, Minas Gerais, Luz, Ribeirão Jorge Grande, 1940'13"S, $45^{\circ} 36^{\prime} 37^{\prime \prime}$ W, 27.IV.2010, H.D.D. Rodrigues col.

Paratypes: 1 macropterous ơ',2 apterous $\%$; glued to pinned paper; Brasil, Minas Gerais, Luz, Ribeirão Jorge Grande, $19^{\circ} 40^{\prime} 13^{\prime \prime}$ S, 4536'37"W, 06.I.2010, H.D.D. Rodrigues col.; 2 apterous $0^{\prime \prime}, 1$ macropterous $\$$, Brasil, Minas Gerais, Luz,

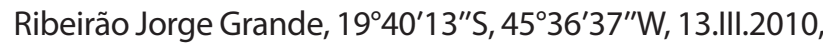
H.D.D. Rodrigues \& G.J.C. Vianna col.

\section{Paravelia bachmanni Rodrigues \& Moreira, 2016}

Paravelia bachmanni Rodrigues \& Moreira, 2016c: 643, 645 , figs. $1-3,10-12,51$.

Holotype: Macropterous on, glued to pinned paper with abdominal segment VIII and genitalia in microvial; Brasil,
São Paulo, Salesópolis, Estação Biológica de Boracéia, in Bromeliaceae, 11.VIII.1961, R.A. Rocha col.

Paratypes: 2 macropterous $q$, glued to pinned paper; same data as holotype. 1 macropteorus $\sigma^{\prime \prime}, 1$ macropterous $\$$, glued to pinned paper; Brasil, Santa Catarina, Santo Amaro da Imperatriz, Hotel Plaza, 82, in Canistrum lindenii, 21.V.2003, J. Steiner \& A. Zillikens. 1 macropterous $\sigma$, 1 macropterous $q$, glued to pinned paper; Brasil, Santa Catarina, Florianópolis, Unidade de Conservação Ambiental Desterro - UCAD, 28.XI.2003, J. Steiner \& A. Zillikens. 1 macropterous ơ", 1 macropterous $\$$, glued to pinned paper; Brasil, Santa Catarina, Santo Amaro da Imperatriz, 402, in Nidularium innocentii, 25.I.2005, J. Steiner \& A. Zillikens. 1 macropterous , glued to pinned paper; Brasil, Santa Catarina, Santo Amaro da Imperatriz, 414, in Nidularium innocentii, 12.V.2005, J. Steiner \& A. Zillikens. 2 macropterous $\sigma^{7}$, 1 macropterous $\$$, glued to pinned paper; Brasil Santa Catarina, Santo Amaro da Imperatriz, 415, in Nidularium innocentii, 12.V.2005, J. Steiner \& A. Zillikens. 1 macropterous $\sigma^{x}$, glued to pinned paper; Brasil, Santa Catarina, Santo Amaro da Imperatriz, 421, in Nidularium innocentii, 05.VII.2005, J. Steiner \& A. Zillikens. 1 macropterous $\sigma^{\prime \prime}, 1$ macropterous $\$$; glued to pinned paper; Brasil, Santa Catarina, Santo Amaro da Imperatriz, 422, in Nidularium innocentii, 05.VII.2005, J. Steiner \& A. Zillikens.

\section{Paravelia biae Spangler, 1989}

Paravelia biae Spangler, 1989: 360-365, figs. 1-8, 15-16.

Holotype: Macropterous ơ, glued to pinned paper with genitalia in microvial; Brazil, Pará, Rio Xingu camp $\left(52^{\circ} 22^{\prime} \mathrm{W}, 03^{\circ} 39^{\prime} \mathrm{S}\right.$ ) Altamira (ca. $\left.60 \mathrm{~km} \mathrm{~s}\right), 10.0 \mathrm{ct} .1986, \mathrm{P}$. Spangler \& O. Flint col.

Paratypes: 6 macropterous $\sigma^{\prime \prime}, 7$ macropterous 9 (19 indicated as allotype), glued to pinned paper; same data as holotype.

\section{Paravelia bilobata Rodrigues et al., 2014}

Paravelia bilobata Rodrigues, Moreira, Nieser, Chen \& Melo in Rodrigues et al., 2014b: 8-10, figs. 4, 6A-F, 21.

Paratype: 1 macropterous $\sigma^{*}$, glued to pinned paper with abdominal segment VIII and genitalia in microvial; Brasil, Mato Grosso, Nova Xavantina, Córrego da Mata, fourth order, $15^{\circ} 01^{\prime} 32^{\prime \prime} \mathrm{S}, 52^{\circ} 26^{\prime} 29^{\prime \prime} \mathrm{W}, 17 . \mathrm{XI} .2005,1788$, H.S.R. Cabette et al.

\section{Paravelia bipunctata Rodrigues et al., 2014}

Paravelia bipunctata Rodrigues, Moreira, Nieser, Chen \& Melo in Rodrigues et al., 2014b: 10-14, figs. 5, 6G-I, 21. 
Paratypes: 1 micropterous $\$$, glued to pinned paper; Brasil, Minas Gerais, Serra do Cipó, [MG-010], km 117, 26.VII.1972, C.G. Froehlich. 1 macropterous , glued to pinned paper; Brasil, Mato Grosso, Nova Xavantina, Córrego Bacaba, ponto 1, 5906, 14²4'03.5"S, $52^{\circ} 21^{\prime} 48.3^{\prime \prime} \mathrm{W}, 01 . V I .2011$, N.F.S. Giehl \& P.V.B. Fonseca. 1 macropterous $\sigma^{\prime}$, glued to pinned paper; Brasil, Mato Grosso, Nova Xavantina, Córrego Bacaba, ponto 3, 6728, $14^{\circ} 43^{\prime} 14.80^{\prime \prime}$ S, 53²1'35.63"W, 29.VI.2012, N.F.S. Giehl \& P.V.B. Fonseca col.

\section{Paravelia bromelicola Rodrigues \& Moreira, 2016}

Paravelia bromelicola Rodrigues \& Moreira, 2016c: 645, 647, figs. 4-5, 13-15, 51.

Holotype: Macropterous $\sigma^{\prime \prime}$, glued to pinned paper with abdominal segment VIII and genitalia in microvial; Brasil, Santa Catarina, Hammonia, tombo 15.550 (70.965), VII.1910, H. Lüderwaldt col.

Paratype: Macropterous $\sigma^{\prime}$, glued to pinned paper with abdominal segment VIII and genitalia in microvial; Brasil, São Paulo, Cubatão, Estação Raiz da Serra, entre folhas de Bromeliaceae, tombo 9.968 (70.318), 30.IX.1907, H. Lüderwaldt col.

Remarks: Unique specimen records 15,550 (holotype) and 9,968 (paratype) are field numbers from Hermann von Lüderwaldt (1865-1934), whereas 70,965 (holotype) and 70,318 (paratype) are likely accession numbers from MZUSP, which are no longer used today.

\section{Paravelia cunhai Rodrigues \& Moreira, 2016}

Paravelia cunhai Rodrigues \& Moreira, 2016b: 184-187, figs. 1-7, 11.

Holotype: Macropterous $\sigma^{*}$, glued to pinned paper with abdominal segment VIII and genitalia in microvial; Brasil, Pará, Vitória do Xingu, comunidade Leonardo da Vinci, poça dentro de caverna, $03^{\circ} 09^{\prime} 07^{\prime \prime} \mathrm{S}, 52^{\circ} 04^{\prime} 30^{\prime \prime} \mathrm{W}$, 08.II.2014, E.J.R. Cunha.

\section{Paravelia digitata Rodrigues \& Moreira, 2016}

Paravelia digitata Rodrigues \& Moreira, 2016c: 647-649, figs. 6-7, 16-18, 31, 51.

Holotype: Macropterous $\sigma^{\prime \prime}$, glued to pinned paper with abdominal segment VIII and genitalia in microvial; Brasil, Bahia, Santa Maria da Vitória, gruta da Bananeira, no date, F. Chaimowicz.

Paratypes: 1 macropterous $\$$, glued to pinned paper; same data as holotype. 3 macropterous $0^{\prime \prime}, 1$ macropterous $\$$, glued to pinned paper; Brasil, Bahia,
Serra do Ramalho, Gruna do Google, 13³7'42.0"S, $43^{\circ} 48^{\prime} 49.0^{\prime \prime} \mathrm{W}, 13 . \mathrm{IX} .2008$, M.E. Bichuette \& R. Rantin. 2 macropterous $0^{\prime \prime}, 1$ macropterous $\uparrow$, glued to pinned paper; Brasil, Bahia, Serra do Ramalho, Gruna Vila

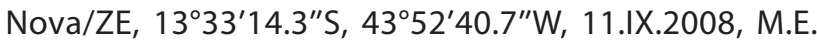
Bichuette \& R. Rantin. 2 macropterous $\$$, glued to pinned paper; Brasil, Bahia, Serra do Ramalho, Gruna

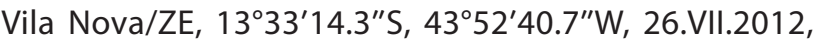
M.E. Bichuette \& J.E. Gallão. 1 macropterous $\$$, glued to pinned paper; Brasil, Bahia, Serra do Ramalho,

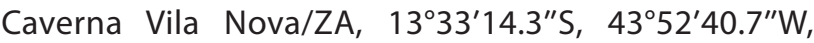
27.VII.2012, M.E. Bichuette \& J.E. Gallão. 1 macropterous $0^{7}, 1$ macropterous $\$$, glued to pinned paper; Brasil, Bahia, Cariranha, Caverna Domingão, 1344'40.7"S, 4349'59.7"W, 27.VII.2012, M.E. Bichuette \& J.E. Gallão. 7 macropterous $\$$, glued to pinned paper; Brasil, Bahia, luiú, Lapa do Baixão-Epígeo, 14²3'08.11"S, 4337'35.00"W, 06.XII.2012, M.E. Bichuette \& J.E. Gallão. 11 macropterous $\sigma^{\prime \prime}, 4$ macropterous $\%$, glued to pinned paper; Brasil, Bahia, Coribe, Gruna do Enfurnado,

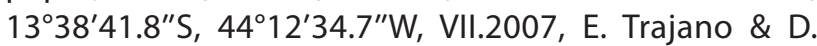
Sansone. 1 macropterous $\$$, glued to pinned paper; Brasil, Bahia, Feira da Mata, Caverna Chico Pernambuco, $13^{\circ} 49^{\prime} 09.0^{\prime \prime}$ S, 440.'14.9"W, 28.VII.2012, M.E. Bichuette \& J.E. Gallão. 1 macropterous $\$$, glued to pinned paper; Brasil, Bahia, São Desidério, Gruta Baixa Fria, $12^{\circ} 22^{\prime} 55.2^{\prime \prime} \mathrm{S}, 44^{\circ} 56^{\prime} 14.4^{\prime \prime} \mathrm{W}, 04 . X I .2008$, M.E. Bichuette, T.L.C. Scatolini \& D.R. Pedroso. 1 macropterous $\sigma^{\prime \prime}, 1$ macropterous $\$$, glued a pinned paper; Brasil, Bahia, Andaraí, Gruna dos Torras, $12^{\circ} 51^{\prime} 41.6^{\prime \prime} \mathrm{S}, 41^{\circ} 18^{\prime} 52.6^{\prime \prime} \mathrm{W}$, 14.VII.2008, M.E. Bichuette, T.L.C. Scatolini \& D.R. Pedroso. 1 macropterous $\%$; glued to pinned paper; Brasil, Rio Grande do Norte, Martins, Gruta Labirinto, $06^{\circ} 03^{\prime} 23^{\prime \prime} \mathrm{S}, 37^{\circ} 53^{\prime} 46^{\prime \prime} \mathrm{W}, 10.1 .2013$, M. Kramer.

\section{Paravelia lacrymosa Rodrigues et al., 2014}

Paravelia lacrymosa Rodrigues, Moreira, Nieser, Chen \& Melo in Rodrigues et al., 2014b: 14-15, figs. 7, 9A, 21.

Holotype: Macropterous $\$$, glued to pinned paper; Brasil, MG [Minas Gerais], Serra [do] Caraça, 1380 m, XI.[1]961, Kloss, Lenko, Martins \& Silva col.

\section{Paravelia luederwaldti Rodrigues \& Moreira, 2016}

Paravelia luederwaldti Rodrigues \& Moreira, 2016c: 649-651, figs. 8-9, 19-21, 51.

Holotype: Brachypterous on, glued to pinned paper with abdominal segment VIII and genitalia in microvial; Brasil, São Paulo, Alto da Serra, Paranapiacaba, 19.334/70.307, 1909, H. Lüderwaldt col.

Paratypes: 4 macropterous $\$$, glued to pinned paper; same data as holotype, except the number: 70.459 , $70.460,70.461,70.462$. 


\section{Paravelia micromaculata Rodrigues et al., 2014}

Paravelia micromaculata Rodrigues, Moreira, Nieser, Chen \& Melo in Rodrigues et al., 2014b: 15-17, figs. 8, 9B, 21.

Holotype: Macropterous $\$$, glued to pinned paper; Brasil, Maranhão, Aldeia Yavaruhu, Rio Gurupi, about $30 \mathrm{~km}$ west of Canindé, isolated trail, forest stream with mud, gravel and leaf litter, 14.II.1966, B. Malkin col.

\section{Paravelia nieseri Moreira \& Barbosa, 2012}

Paravelia nieseri Moreira \& Barbosa, 2012: 61-63, figs. 10-18.

Holotype: Macropterous ơ', glued to pinned paper; Serra [do] Caraça, 1.380 m, MG [Minas Gerais] - Brasil, XI.[1]961, Kloss, Lenko, Martins \& Silva col.

Paratypes: 2 macropterous $\sigma^{*}$, glued to pinned paper with abdominal segment VIII and genitalia in microvial; same data as holotype. 1 macropterous $\$$, glued to pinned paper; [Brasil] S.[erra do] Caraça (Engenho), 800 m, Minas [Gerais] XI.[1]961, Kloss, Lenko, Martins \& Silva col.

\section{Paravelia ornata Rodrigues et al., 2014}

Paravelia ornata Rodrigues, Moreira, Nieser, Chen \& Melo in Rodrigues et al., 2014b: 17-19, figs. 10, 12A-D, 21.

Holotype: Macropterous $\sigma^{*}$, glued to pinned paper; [Brasil], Tapuruquara, Rio Negro, AM[azonas], 26-27. XI.1962, J. Bechyné col.

Paratypes: 3 macropterous ơ, 2 macropterous $\$$, glued to pinned paper, a male with abdominal segment VIII and genitalia in microvial; Barcelos AM[azonas], Brasil, 18-22.II.1963, J. Bechyné col.

Remarks: In the original description, the authors specified geographical coordinates for the two localities mentioned above.

\section{Rhagovelia ochroischion Nieser \& Melo, 1997}

Rhagovelia ochroischion Nieser \& Melo, 1997: 105, 107, fig. 121.

Lectotype: Apterous o", glued to pinned paper; Brasil, M.G. [Minas Gerais], Carmen d.[o] R.[io] Claro, Córrego do Castelhano, lowland type stream, 13.III.[19]94, Nieser col.

Paralectotype: 1 apterous $\&$, glued to pinned paper; same data as holotype.
Remarks: Nemésio \& Melo (2012) made the lectotype designation. The nomenclatural history of this species can be found in Moreira et al. (2010) and Nemésio \& Melo (2012).

\section{Rhagovelia sbolos Nieser \& Melo, 1997}

Rhagovelia sbolos Nieser \& Melo, 1997: 105, 107, figs. 122-123.

Lectotype: Apterous $\$$, glued to pinned paper; Brasil, M.G. [Minas Gerais], Nova Lima, São Seb.[astião das] Ag.[uas] Claras, Ribão [Ribeirão] Marumbé, 01.IIl.1994, Nieser col.

Paralectotype: Apterous $\sigma^{n}$, glued to pinned paper; same data as holotype.

Remarks: Nemésio \& Melo (2012) made the lectotype designation. The nomenclatural history of this species can be found in Moreira et al. (2010) and Nemésio \& Melo (2012).

\section{Rhagovelia trianguloides Nieser \& Melo, 1997}

Rhagovelia trianguloides Nieser \& Melo, 1997: 109.

Lectotype: Apterous $\sigma^{x}$, double-mounted on minuten pin; Brasil, MG [Minas Gerais], Bel.[o] Horz[Horizonte], Nova Lima, [São Sebastião das] A.[guas] Claras, Ribão [Ribeirão] Marumbé, N9215, \#25, XI.1992, N. Nieser col.

Remarks: Nemésio \& Melo (2012) made the lectotype designation. The nomenclatural history of this species can be found in Moreira et al. (2010) and Nemésio \& Melo (2012).

\section{Rhagovelia turmalis Nieser \& Melo, 1997}

Rhagovelia turmalis Nieser \& Melo, 1997: 104, 107.

Lectotype: Apterous ơ, glued to pinned paper; Brasil, M.G. [Minas Gerais], Sra[Serra do] Cipó, córrego do Palacio, km 120 \#1992, N9225, 05.XII, N. Nieser col.

Paralectotype: Apterous $\$$; glued to pinned paper; same data as holotype.

Remarks: Nemésio \& Melo (2012) made the lectotype designation. The nomenclatural history of this species can be found in Moreira et al. (2010) and Nemésio \& Melo (2012).

\section{Infraorder Nepomorpha Popov, 1971 Naucoridae Leach, 1815} Carvalhoiella acuminata Rodrigues \& Sites, 2016

Carvalhoiella acuminata Rodrigues \& Sites in Rodrigues et al., 2016: 578-581, figs. 1-6, 32. 
Holotype: Macropterous $\$$, pinned; Brasil, Mato Grosso, Nova Xavantina, waterfall at Córrego Chupador, 18.IX.2010, H. Cabette et al. col.

Paratypes: 2 macropterous $\sigma^{7}$, pinned; same data as holotype. 1 macropterous ơ", specimen pinned; Brasil, Mato Grosso, Nova Xavantina, waterfall at Córrego Chupador, 29.IV.2011, H. Cabette et al. col. 1 macropterous ơ, 1 macropterous $\$$, pinned; Brasil, Mato Grosso, Nova Xavantina, waterfall at Córrego Buriti, 14 $45^{\prime} 04.1^{\prime \prime} \mathrm{S}, 52^{\circ} 33^{\prime} 11.0^{\prime \prime} \mathrm{W}$, 27.IV.2011, H. Cabette et al. col. 1 macropterous o", pinned; Brasil, Mato Grosso, Nova Xavantina, waterfall at Córrego Buriti, 14²4'04.1"S, 52³3'11.0"W, 19.IX.2010, H. Cabette et al. col. 2 macropterous o", pinned; Brasil, Mato Grosso, Nova Xavantina, waterfall at Córrego Duílio, 17.IX.2010, $\mathrm{H}$. Cabette et al. col. 1 macropterous ${ }_{\text {, }}$ pinned; Brasil, Mato Grosso, Nova Xavantina, waterfall at Córrego Duílio, downstream, 14²8'18.8'S, 52³0'10.7"W, 01.V.2011, H. Cabette et al. col. 1 macropterous $\sigma^{\prime \prime}$, pinned; Brasil, Mato Grosso, Nova Xavantina, waterfall at Córrego Duílio, downstream, $14^{\circ} 48^{\prime} 18.8^{\prime \prime} \mathrm{S}, 52^{\circ} 30^{\prime} 10.7^{\prime \prime} \mathrm{W}$, 01.V.2011, N.F.S. Giehl col.

\section{Carvalhoiella helenae Rodrigues \& Sites, 2016}

Carvalhoiella helenae Rodrigues \& Sites in Rodrigues et al., 2016: 581, 582-585, figs. 13-18, 32.

Holotype: Macropterous $\$$, pinned; Brasil, Mato Grosso, Nova Xavantina, Córrego Buriti, upstream, 1445'04.1"S, 52³3'11.0"W, 27.IV.2011, H. Cabette et al. col.

Paratype: 1 macropterous $\sigma^{\prime \prime}$, pinned with abdominal segments VI-VIII and genitalia in microvial; same data as holotype.

\section{Nepidae Latreille, 1802 Curicta granulosa De Carlo, 1951}

Curicta granulosa De Carlo, 1951: 411-412, figs. 35-38.

Holotype: $\sigma^{x}$, pinned; [Brazil] Amazonas, Mana'os [Manaus], 1899, Bicego leg.

Allotype: $\$$, pinned; same data as holotype, except 19336.

Paratype: 19 , pinned; without label of locality.

Remarks: The paratype has no locality label; however, according to the original description, all specimens in type series were collected on the same site.

\section{Ranatra lanei De Carlo, 1946}

Ranatra lanei De Carlo, 1946: 21-22, fig. 72.
Holotype: $\sigma^{\pi}$, pinned; [Brazil], Rio de Janeiro, Campo Bello, IV-[19]06.

Allotype: $\$$, pinned; same data as holotype.

Remarks: In the original description, the author mentions that the collector of the type series is "Luderbald". That person is probably Hermann von Lüderwaldt (1865-1934), technician of the Museu Paulista at the time. The type-locality is currently part of the Itatiaia National Park.

\section{Ranatra neivai De Carlo, 1946}

Ranatra neivai De Carlo, 1946: 31-32, fig. 82.

Holotype: ơ, pinned; [Brazil], São Paulo, Capital, X.1937, R. Araujo col.

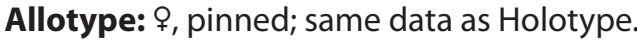

\section{Notonectidae Latreille, 1802 Buenoa koina Nieser \& Pelli, 1994}

Buenoa koina Nieser \& Pelli, 1994: 3-4, figs. 8-10.

Holotype: Macropterous $\sigma^{\pi}$, pinned with left fore leg glued to a paper; Brasil, MG [Minas Gerais], Serra da Moeda, km 34 da BR-135, Nova Lima, 20.08.[19]72, H.N. Espínola col.

Allotype: Macropterous $q$, pinned; same data as holotype.

\section{Buenoa konta Nieser \& Pelli, 1994}

Buenoa konta Nieser \& Pelli, 1994: 1-3, figs. 1-4.

Paratypes: 1 brachypterous ơ, 1 brachypterous 9 ; glued to pinned paper; Brasil, M.G. [Minas Gerais], Lagoa Santa, Lagoa Olhos D’Água, 05.09.[19]91, Pelli, A. col.

\section{Potamocoridae Usinger, 1941 Coleopterocoris nelsoni Longo et al., 2005}

Coleopterocoris nelsoni Longo, Ribeiro \& Nessimian, 2005: 41-43, figs. 3-8.

Paratypes: 3 brachypterous $\sigma^{\prime \prime}, 7$ brachypterous $\%$, in ethanol; Brasil, Rio de Janeiro, Macaé, Rio Macaé, 29.VIII.2003, N. Ferreira-Jr leg.

Remarks: The genus Coleopterocoris Hungerford, 1942 is currently junior synonym of Potamocoris 1941 (Polhemus \& Carrie, 2013). Therefore, the current status of this species is Potamocoris nelsoni (Longo et al., 2005). In the orig- 
inal description, the authors designated 4 males and 6 female paratypes to MZUSP but these are actually 3 males and 7 females.

\section{Infraorder Pentatomomorpha Leston et al., 1954 Aradidae Spinola, 1837 \\ Proxius (Neoproxius) amazonicus Kormilev, 1976}

Proxius (Neoproxius) amazonicus Kormilev, 1976: 739-740, figs. 4-5.

Holotype: + , glued to pinned paper; Brazil, Amazonas, 5 km W Cacau Pereira, across Rio Negro from Manaus, 120 m, July[VII] 24, 1973, R.T. Schuh col.

\section{Aneurus carioca Kormilev, 1968}

Aneurus carioca Kormilev, 1968: 48-49, figs. 1-2.

Holotype: $\$$, pinned; [Brazil], Rio de Janeiro, VIII.1952, Ary Vulcano col.

Remarks: In the original description, the collection year is 1962, however the label specifies 1952. The current status of the species is Iralunelus carioca (Kormilev, 1968).

\section{Calisius pulcher Kormilev, 1976}

Calisius pulcher Kormilev, 1976: 737-739, fig. 3.

Holotype: $\sigma^{\prime}$, glued to pinned paper; Brazil, Minas Geraes [Gerais], Pedra Azul, XI.1971, Seabra and Oliveira col.

\section{Mezira gracilis Kormilev, 1968}

Mezira gracilis Kormilev, 1968: 52-53, figs. 5-6.

Holotype: $\sigma^{\top}$, glued to pinned paper; Brasil, Amazonas, Manaus, VIII.1962 K. Lenko col.

Paratype: $10^{n}$, glued to pinned paper; same data as holotype.

\section{Mezira pilosa Kormilev, 1968}

Mezira pilosa Kormilev, 1968: 51-52, figs. 3-4.

Holotype: $\sigma^{n}$, glued to pinned paper; Mexico, E[sta]do. Mexico, Bosenchebe, 01.III.1964, P. Reyes C. col.

\section{Mezira timida Kormilev, 1968}

Mezira timida Kormilev, 1968: 53-54, fig. 7
Holotype: $\$$, glued to pinned paper; [Brazil], MT [Mato Grosso], Utiariti, Rio Papagaio, XI.1966, Lenko and Pereira col.

Paratype: 19, glued to pinned paper; Brasil, Mato Grosso, Corumbá, Serra do Urucum, 23.30/XI.1960, K. Lenko col.

\section{Coreidae Leach, 1815}

Althos terminalis Brailovsky, 1990

Althos terminalis Brailovsky, 1990: 76-79, figs. 13-15, 67, $68,85,86,106,124,239$.

Paratypes: 1\%, 10", pinned; Brasil, MG [Minas Gerais], Serra [do] Caraça 1380 m, XI.[1]961, Kloss, Lenko, Martins and Silva col. 10', pinned; Brasil, São Paulo, Osasco, 09.XI.1956, M.A. Vulcano col.

Remarks: In the original description, the specimens from Minas Gerais are reported to occur in the state of Mato Grosso. This was probably a misunderstanding of the initials written on the label - MG refers to Minas Gerais.

\section{Paryphes pullulus Brailovsky, 1986}

Paryphes pullulus Brailovsky, 1986a: 108-110, figs. 5, 6, 17, $18,23-25$.

Paratypes: 29, pinned; [Brazil], Amaz[onas]., Manaus, Rio Negro, 14.VII.1927, J.F. Zikán col.

\section{Stenoprasia abortiva Brailovsky, 1986}

Stenoprasia abortiva Brailovsky, 1986b: 457, fig. 10.

Holotype: $\sigma^{*}$, pinned; [Brazil], Est[ado]. do Amazonas, Rio Juruá, 1901, E. Garbe col.

\section{Sundarus volutatorius Brailovsky, 1988}

Sundarus volutatorius Brailovsky, 1988: 578-580, fig. 49.

Paratype: 19, pinned; [Brazil], Amaz.[onas], Pará, Santarém, R[io] Juruá 1901, E. Garbe col.

\section{Sundarus zonatus Brailovsky, 1988}

Sundarus zonatus Brailovsky, 1988: 593-596, figs. 43, 57.

Paratypes: $10^{\pi}$, pinned; Peru, Satipo, II.1938, P. Paprizyck col. 10", pinned; Peru, Satipo, Huancayo, I. 1944, P. Paprizyck col. 10", pinned; Peru, Satipo, Huancayo, II.1944, P. Paprizyck col. 19, pinned; Peru, Satipo, Huancayo, III.1944, P. Paprizyck col. 10', pinned; Peru, Satipo, Huancayo, II.1944, P. Paprizyck col. 


\section{Pentatomidae Leach, 1815 Callostethus flavolineatus Fernandes \& Grazia, 2011}

Callostethus flavolineatus Fernandes \& Grazia in Fernandes et al., 2011: 57-59, figs. 5-7, 9.

Holotype: $\sigma^{\prime}$, pinned with genitalia in a microvial; [Brazil], Ter.[ritório do] Amapá, Rio Aniconi, 16.VI.[19]59, J. Lane col.

\section{Catulona lucida Campos \& Grazia, 2004}

Catulona lucida Campos \& Grazia in Campos et al., 2004: 4-7, figs. 3-9.

Paratypes: 30', 19, pinned; [Brazil], São Paulo, Batatais, 12-43.XII-1943, Padre P. col.

\section{Cyrtocoris egeris Packauskas \& Schaefer, 1998}

Cyrtocoris egeris Packauskas \& Schaefer, 1998: 373-375, figs. 3, 6-7, 15.

Paratypes: 19, pinned; Ecuad[or], Balzapamba, no date, R. Haensch S. col. 10', pinned; Brasil, SP [São Paulo], S[ão]. Sebastião, Barra do Una, 08.VII.[1]961, K. Lenko col. 1\%, pinned; Brasil, São Paulo, Barueri, 24.IV.1955, K. Lenko col. 19, pinned; Brasil, São Paulo, Barueri, K. Lenko 26.I.1955, K. Lenko col. 19, pinned; Brasil, São Paulo, Barueri, K. Lenko 31.XII.1954, K. Lenko col. 1\%, pinned; Brasil, SP [São Paulo], Mogi Guaçu, Faz[enda]. Campinas, XII.29-31.1969, JM e BA Campbell col. 1\%, pinned; [Brazil], R[io de] Janeiro, Corcovado, 01.XII.1961 Warner col. 19, pinned; Brasil, E[spirito]. Santo, Corrego do Itá, XI-XII-1956, W. Zikan col. 4ㅇ, 30", pinned; Brasil, Goias, Jataí, Faz[enda]. Aceiro, X.1962, Exp[edição]. Dep[artamento]. Zool[ogia]. 19, pinned; Brasil, São Paulo, Itu, Faz[enda]. Pau D'alho, XII.1956, U. Martins col. 19, pinned; [Brazil], Goiás, Anapolis, V.1939, P.J. Ribeiro col. 1\%, pinned; [Brazil], [Minas Gerais], Monte Alegre, Sta. [Santa] Maria, Alt[itude]. 1.100 m[e]t[er]s. 24-30.XI.1942, F. Lane col. 39, 10', pinned; BR [Brazil], Rio de Janeiro, Nova Friburgo, XII. 1976, Mury, Gred \& Guimarães col. 19, pinned; BR [Brazil], Rio de Janeiro, Nova Friburgo, XII.1980, Mury, Gred \& Guimarães. 19, pinned; [Brazil], RJ [Rio de Janeiro], Nova Friburgo, XII.1961, J.H. Guimarães col. 19, pinned; [Brazil], RJ [Rio de Janeiro], Nova Friburgo, XII.1975, J.H. Guimarães col. 19, 10', pinned; Brasil, SC [Santa Catarina], Nova Teutonia, III.1966, F. Plaumann col. 19, 10, pinned; [Brazil], E.S [Espirito Santo], Linhares, Parque Sooretama, 17-27.X.1962, F.S. Pereira col. 3\%, pinned; Brasil, S.P. [São Paulo], Rio Claro, 18.I.1962, Biasi, Reichardt col. 1\%, pinned; [Brazil], São Paulo, Cidade Jardim, 23.Il.1941 F. Lane col. 1ㅇ, pinned; [Brazil], São Paulo, Osasco, 15.IV.1938, F. Lane col. 19, pinned; [Brazil], S[ão]. Paulo, S[erra]. Negra, Sítio B[oa]. Vista, 20.III.1943, Lima col. 2\%, 30', pinned; [Brazil], S.[ão]. Paulo, Serra Negra, Sítio Boa Vista, 20.III.1943, Lima col.
19, pinned; [Brazil], S.[ão]. P[aulo]., Serra Negra, Sítio Boa Vista, 20.III.1943, Lima col.

\section{Euschistus (Mitripus) hansi Grazia, 1987}

Euschistus (Mitripus) hansi Grazia, 1987: 83-87, figs. 1-9.

Holotype: $\sigma^{\pi}$, pinned; [Brazil], R.G.S [Rio Grande do Sul], S[ão]. F[rancisco de]. Paula, Inst[ituto]. Nac[ional do]. Pinho, 22.I.1959, Pereira, Ditadi, Petersen, Meyrer, Volkmer col.

Paratypes: 20", pinned; [Brazil], R.G.S [Rio Grande do Sul], S[ão]. F[rancisco de]. Paula, Inst[ituto]. Nac[ional do]. Pinho, 20.XII.1959, Pereira, Ditadi, Petersen, Meyrer, Volkmer col. 19, pinned; [Brazil], R.G.S [Rio Grande do Sul], S[ão]. F[rancisco de]. Paula, Inst[ituto]. Nac[ional do]. Pinho, 21.XII.1959. Petersen col. 1\%, pinned; BR[azil], SP [São Paulo], Campinas, X.[19]76, S. Pereira col. 19, pinned; BR[asil], SP [São Paulo], Jundiaí, 08.X.1976, B.C. Lopes col.

Remarks: Adustonotus hansi (Grazia, 1987) is currently the valid name of Euschistus (Mitripus) hansi Grazia, 1987.

\section{Euschistus (Mitripus) paranticus Grazia, 1987}

Euschistus (Mitripus) paranticus Grazia, 1987: 87-88, figs. 10-19.

Holotype: $\sigma^{\prime}$, pinned; Brasil, RS [Rio Grande do Sul], Pq. [Parque] Est. [Estadual do] Turvo, 10.I.1982, S.L. Bonatto Col.

Remarks: Adustonotus paranticus (Grazia, 1987) is currently the valid name for Euschistus (Mitripus) paranticus Grazia, 1987.

\section{Dichelops avilapiresi Grazia, 1978}

Dichelops avilapiresi Grazia, 1978: 22-23, figs. 1-9, 126.

Holotype: \$, pinned; Brasil, Paraná, Ponta Grossa, XII. [19]38, Camargo col.

Paratypes: 19, pinned; [Brazil], Paiol [Grande], 03.II.1948 M. Gordinho col. 10', pinned; Brasil, M[inas Gerais], IX.1962 F.S. Pereira col.

\section{Dichelops miriamae Grazia, 1978}

Dichelops miriamae Grazia, 1978: 36-37, figs. 28-36, 126.

Paratypes: $20^{\pi}, 19$, pinned; no locality data.

Remarks: In the original description, the author states that specimens were collected by R. von Ihering in 1933. In addition to the paratype label, the specimens bear 
a previous identification label (D. leucostigma as determined by L. Buckup).

\section{Dichelops phoenix Grazia, 1978}

Dichelops phoenix Grazia, 1978: 80-81, figs. 102-111, 126.

Paratypes: 1\%, pinned; Brasil, GO [Goias], Rib. Vãozinho, 12.ll.1962, J. Bechyné col. 10\%, pinned; [Brazil], Goias, Campinas, Deze[embro] [XII] 1935, Spitz col. 10", pinned with genitalia in a microvial; [Brazil], Goias, Campinas, Deze[embro] [XII] 1935, Spitz col.

\section{Dichelops pradoi Grazia, 1978}

Dichelops pradoi Grazia, 1978: 52-53, figs. 55-63, 126.

Paratype: 19, pinned; Brasil, Mato grosso, Corumbá, Serra do Urucum, 02.XII.1960 R. Lenko col.

\section{Dichelops saltensis Grazia, 1978}

Dichelops saltensis Grazia, 1978: 62-63, figs. 73-91, 126.

Holotype: $\sigma^{2}$, pinned; Argentina. P[rovin]cia. [de] Salta, Pocitos I. 1959, A. Martinz col.

Paratypes: 3\%, pinned; same data as holotype.

Remarks: In the original description, there are one male and two females from Argentina, and one male from Brazil, deposited at MZUSP.

\section{Dinocoris nigrodecoratus Becker \& Grazia, 1985}

Dinocoris nigrodecoratus Becker \& Grazia, 1985: 105-106, figs. $3,16,29,50,51,67,83,90$.

Holotype: \&, pinned; [Brasil], PA [Pará], Santarém, Taperinha, 29.XII.1967-09.I.1968, Exp[edição] Perm[amente]. Amaz[onica].

\section{Dinocoris reticulatus Becker \& Grazia, 1985}

Dinocoris reticulatus Becker \& Grazia, 1985: 99-100, figs. 1, $7,20,33,43,58$.

Holotype: $\sigma^{x}$, pinned with genitalia in a microvial; [Brazil], [Minas Gerais], Lagoa Santa, 28.I.1963, N. Papavero col.

\section{Edessa rufodorsata Silva et al., 2006}

Edessa rufodorsata Silva, Fernandes \& Grazia, 2006: 356, figs. 14, 26, 38-39, 52
Paratype: $10^{\pi}$, pinned with genitalia in a microvial; Brasil, Mato Grosso, Barra do Tapirapé, 14.XII.1964, R. Malkin col.

\section{Edessa virididorsata Silva et al., 2006}

Edessa virididorsata Silva, Fernandes \& Grazia, 2006: 356-361, figs. 15, 27, 40-41, 53.

Paratypes: 10', pinned; BRA[zil]. SP [São Paulo], São Carlos, Fazenda Canchim, Cerrado 23.X.1993, N.W. Perioto col. 10', 19, pinned; [Brazil], MT [Mato Grosso], Utiariti, Rio Papagaio, 21-31.X.1966, Lenko and Pereira col.

\section{Euschistus longicornis Hildebrand \& Grazia, 1983}

Euschistus longicornis Hildebrand \& Grazia, 1983: 82-84, figs. 1-10.

Holotype: $\sigma^{\pi}$, Brasil, Mato Grosso, Barra do Tapirapé, 16-26.I.1964, R. Malkins col.

\section{Galedanta pulchra Grazia, 1967}

Galedanta pulchra Grazia, 1967: 49-51, figs. 1, 3, 7-9.

Holotype: $10^{x}$, pinned with genitalia in a microvial; Brasil, Paraná, Ponta Grossa, no date Camargo col.

Remarks: According to the original description, the holoype is deposited at the Museu Rio-grandense de Ciências Naturais (MRCN) and was collected in XII. [19]38.

\section{Hypantracos ditarsus Grazia \& Campos, 1996}

Hypantracos ditarsus Grazia \& Campos, 1996: 16-19, figs. 2, 4, 9-12, 16-18, 20.

Holotype: $\sigma^{\prime}$, pinned with genitalia in a microvial; Brasil, PA [Pará], Santarém, 04.v.1967, Exp[edição]. Perm[anente]. Amaz[onica].

\section{Lincus anulatus Rolston, 1983}

Lincus anulatus Rolston, 1983: 22-24, figs. 45-48.

Paratypes: 19, pinned; Brasil, PA [Pará], S[an]ta. Isabel do Pará, 30.III.1962, J. Bechyné col. 10", pinned; [Brazil], Amapá, Igarapé Sucuriju, Série Navio, no date, Bicelli col. 10َ' pinned; Brasil, Amazonas, Benjamin Constant, 18-28. IX.1962, K. Lenko col.

\section{Lincus cropius Rolston, 1983}

Lincus cropius Rolston, 1983: 12-13, figs. 12-15. 
Holotype: ơ', pinned; Brasil, Amapá, Rio Pelioca, 04.VIII.1957, J. Lane col.

\section{Neotibilils peruana Grazia \& Barcellos, 1994}

Neotibilils peruana Grazia \& Barcellos, 1994: 76-77, figs. 58, 65, 77-78, 86, 96, 100.

Holotype: $\sigma^{\prime \prime}$, pinned with genitalia in a microvial; Peru, Pucallpa, Rio Ucayali 200 m, Jan [1] 1960, Dirings col.

\section{Pallantia macunaima Grazia, 1980}

Pallantia macunaima Grazia, 1980: 26-27, figs. 28-36.

Paratypes: 19, pinned; Brasil, Minas Gerais, Sete Lagoas, III.1963, F. Werner, U. Martins, L. Silva col. 19, pinned; [Brazil], MT [Mato Grosso], Três Lagoas, Água Tirada [stream], 15-30.V.[19]64, Exp[edição]. Dep[artamento]. Zool.[ogia].

\section{Paralincus silvae Rolston, 1983}

Paralincus silvae Rolston, 1983: 185-187, figs. 2-5.

Paratype: 10', pinned; [Brazil], Ter.[ritório do] Amapá. Rio Amapari 16.VI.[19]35, J. Lane col.

\section{Paratibraca infuscata Campos \& Grazia, 1995}

Paratibraca infuscata Campos \& Grazia, 1995: 165-170, figs. 1-15.

Paratypes: $10^{\pi}$, pinned with genitalia in a microvial; [Brazil], AM [Amazonas], Ilha do Careiro, Lago do Rei, 12.IV.1967, Exp[edição] Perm[amente]. Am[a]z.[onica]. $10^{\prime \prime}$, pinned with genitalia glued to pinned paper; [Brazil], Santos, S[ão]. Paulo, Estrada velha [Caminhos do Mar Park] III.[19]57, Werner col.

\section{Phalaecus nigromaculatus Grazia, 1983}

Phalaecus nigromaculatus Grazia, 1983: 185-186, figs. 23-31.

Paratype: 19 , glued to pinned paper with genitalia in a microvial; Brasil, Terr.[itório do] Amapá, Rio Amapari, 8. VIII. 1959, J. Lane col.

\section{Rio indistincus Fortes \& Grazia, 2000}

Rio indistincus Fortes \& Grazia, 2000: 81-83, figs. 8, 21, 34, $45-46,55-56,65,76,86-87,114$
Paratypes: 2\%, pinned; Brasil, GO [Goias], Jataí Faz[enda]. Nova Orlandia, I.1964, Martins, Morgante \& Silva col.

\section{Sedia robusta Fortes \& Grazia, 2005}

Sedia robusta Fortes \& Grazia, 2005: 324-326, figs. 5, 22, $76,90,107,113$.

Paratypes: 19, pinned; [Brazil], S. [Santa] Catarina, Brusque, XII.[19]57, J. Leme col. 19, pinned; [Brazil], Est. [tação]. Bio.[lógica] Boraceia, Salesópolis, S.P. [São Paulo], 12.II.1963, L. Silva \& H. Reichardt col.

\section{Serdia indistincta Fortes \& Grazia, 2005}

Serdia indistincta Fortes \& Grazia, 2005: 310-312, figs. 3, $20,37,52,69,84$.

Paratype: 1ㅇ, pinned; S.[São] Paulo 13.XII.1916, Costa Lima col.

\section{Tibilis apposita Barcellos \& Grazia, 1993}

Tibilis apposita Barcellos \& Grazia, 1993: 188-191, figs. 2-5, $14,17,20,23,28$.

Paratypes: $10^{*}$, pinned with genitalia in a microvial; [Brazil], Caraguatatuba SP [São Paulo]. Res. Flor. - 40 m, 02.IV.[1]962, Martins Reichardt and Silva col. 1\%, pinned; [Brazil], E[stado]. Rio [de Janeiro], Itatiaia, Maromba, 26.12[XII].1952, C. Leite, Seabra \& Zikan col.

\section{Tibilis riograndensis Barcellos \& Grazia, 1993}

Tibilis riograndensis Barcellos \& Grazia, 1993: 192-195, figs. $6-9,15,18,21,25,30$.

Paratypes: 1\%, 10", pinned; [Brazil], RS [Rio Grande do Sul], São Leopoldo, 01.X.1986, C.J. Becker col.

\section{Rhyparochromidae Amyot \& Serville, 1843 Paisana lydiae Dellapé, 2008}

Paisana lydiae Dellapé, 2008: 24, 25, fig. 3, 16-20.

Holotype: $\sigma^{\star}$, one leg glued to pinned paper with genitalia in a microvial; Brasil, MG [Minas Gerais], Serra Caraça, 1390 m, XI.[1]9611, Kloss, Lenko, Martins \& Silva col.

Paratype: 19 , glued to pinned paper; same data as holotype.

Remarks: In the original description, two females are designated as paratypes, however, only one was found at MZUSP. 


\section{ACKNOWLEDGMENTS}

We are grateful to Marcelo Duarte, Ana Vasquez and Carlos Campaner (MZUSP) granting us access to the collection and the museum facilities. We thank the editor, two anonymous reviewers and Julia Meyer for offering constructive suggestions. CFS thanks the CNPq Research Fellowship program (Proc. n. 313214/2017-4). This study was financed in part by the Coordenação de Aperfeiçoamento de Pessoal de Nível Superior - Brasil (CAPES) - Finance Code 001 (scholarship to RC), and by the Fundação de Amparo à Pesquisa do Estado de São Paulo, FAPESP) (Proc. n. 13/16654-0, scholarship to HDDR; Proc. n. 15/50322-0, regular grant to CFS).

\section{REFERENCES}

Aristizábal-García, H.; Floriano, C.F.; Moreira, F.F. \& Bispo, C.P. 2015. New species and notes on Microveliinae from northern South America (Hemiptera: Heteroptera:Veliidae). Zootaxa, 3980(4): 591-596.

Barcellos, A. \& Grazia, J. 1993. Revisão de Tibilis Stål, 1860 (Heteroptera, Pentatomini). Anais da Sociedade Entomológica do Brasil, 22: 183-208.

Becker, M. \& Grazia, J. 1985. Revisão do gênero Dinocoris Burmeister, 1835 (Heteroptera, Pentatomidae, Discocephalinae). Revista Brasileira de Zoologia, 3: 65-108.

Brailovsky, H. 1986a. Nuevos datos distribucionales y descripcion de tres nuevas especies del genero Paryphes Burmeister (Hemiptera-HeteropteraCoreidae-Coreini). Anales del Instituto de Biología, Universidad Nacional Autónoma de México, Serie Zoología, 56: 101-128.

Brailovsky, H. 1986b. Revision del genero Stenoprasia Horvath descripcion de dos especies nuevas (Hemiptera-Heteroptera-Coreidae-Coreini). Anales del Instituto de Biología, Universidad Nacional Autónoma de México, Serie Zoología, 56: 453-464.

Brailovsky, H. 1988. Revision del genero Sundarus Amyot-Serville (HemipteraCoreidae-Coreini) para el continente Americano. Anales del Instituto de Biología, Universidad Nacional Autónoma de México, Serie Zoología, 58:561-622.

Brailovsky, H. 1990. Revision del complejo “Althos" con descripción de géneros nuevos y especies nuevas (Hemiptera: Heteroptera: Coreidae: Coreini). Publicaciones especiales del Instituto de Biología, 5: 1-156.

Campos, L.A. \& Grazia, J. 1995. Paratibraca, um novo gênero de Pentatomini (Heteroptera, Pentatomidae). Iheringia, Série Zoologia, (79): 163-171.

Campos, L.A. \& Grazia, J. 2004. In: Campos, L.A.; Grazia, J. \& Greve, C. 2004. Notes on Catulona Rolston, 1992, and the description of a new species from Brazil (Hemiptera, Pentatomidae, Discocephalinae). Zootaxa, 404(1): 1-7.

Carvalho, J.C.M. 1989. Mirideos neotropicais, CCCIV: novos gêneros e espécies do Brasil (Hemiptera). Revista Brasileira de Biologia, 49: 443-460.

Carvalho, J.C.M. 1990. Mirideos neotropicais, CCCVII: novas espécies da Argentina e Bolivia (Hemiptera). Revista Brasileira de Entomologia, 34: 445-452.

Carvalho, J.C.M. \& Costa, L.A.A. 1989. Mirideos neotropicais, CCCV: espécies sul-americanas do gênero Proba Distant (Hemiptera). Anais da Academia Brasileira de Ciências, 60: 333-346.

Carvalho, J.C.M. \& Costa, L.A.A. 1991. Mirideos Neotropicais, CCCXLVIII: Gêneros e Espécies Novos da América Central e América do Sul, com nota sobre Prepops oranensis Carvalho \& Capintero, 1987 (Hemiptera). Anais da Academia Brasileira de Ciências, 63: 43-57.

Carvalho, J.C.M. \& Costa, L.A.A. 1992. Descriptions of new species of Notocyrtus Burmeister, 1835 (Hemiptera-Heteroptera, Reduviidae, Harpactorinae). Anais da Academia Brasileira de Ciências, 64: 71-78.

Coscarón, M.D.C. 1983. Revision del genero Rasahus (Insecta, Heteroptera, Reduviidae). Universidad Nacional de La Plata, 75-138.
Coscarón, M.D.C. \& Carpinteiro, D.L. 1993. Revision of the genus Melanolestes Stål (Heteroptera: Reduviidae, Peiratinae). Entomologica Scandinavica, 24:361-381.

Costa Lima, A.M.C. 1940. Novo hemiptero Reduviidae da subfamília Vesciinae. Arquivos de Zoologia do Estado de São Paulo, 1: 485-490.

De Carlo, J.A. 1946. Los Ranatridae de Sud America. Anales del Museo Argentino de Ciencias Naturales, 161: 1-38.

De Carlo, J.A. 1951. Nepidos de América (Hemiptera: Nepidae). Revista del Instituto Nacional de Investigacion de las Ciencias Naturales, 1(9): 385-421.

Dellapé, P.M. 2008. Paisana: A new genus of Neotropical Rhyparochromidae (Hemiptera: Heteroptera: Lygaeoidea) to accommodate Neopamera brachialis (Stål) and four new species. Zootaxa, 1958: 17-30.

Drake, C.J. 1928. Concerning some tingitidae from Argentina with descriptions of five new species. Physis: Revista de la Sociedad Argentina de Ciencias Naturales, 9: 72-76.

Drake, C.J. 1940. New American Teleonemia (Hemip.: Tingitidae). Revista Chilena de História Natural, 44: 242-243.

Drake, C.J. \& Hambleton, E.J. 1934. Brazilian Tingitidae (Hemiptera) (Part I). Revista de Entomologia, Rio de Janeiro, 4: 435-451.

Drake, C.J. \& Hambleton, E.J. 1935. New Brazilian Tingitidae (Hemiptera) (Part II). Archivos do Instituto Biologico, 6: 141-154.

Drake, C.J. \& Hambleton, E.J. 1938. Concerning Brazilian Tingitidae (Hemiptera) (Part III). Revista Entomologica, 8: 44-68.

Drake, C.J. \& Hambleton, E.J. 1946. New species and new genera of American Tingidae (Hemiptera). Proceedings of the Biological Society of Washington, 59: 9-16.

Drake, C.J. \& Poor, M.E. 1938. Nine new American Tingitidae (Hemiptera). Bulletin of the Brooklyn Entomological Society, 33: 28-34.

Drake, C.J. \& Poor, M.E. 1940. Six new South American Tingitidae (Hemiptera). Revista de Entomologia, 11:226-231.

Evangelista, 0.; Santos, G.I.M. \& Lamas, C.E. 2014. An annotated catalogue of the Membracidae types in the Museu de Zoologia da Universidade de São Paulo, Brazil (Hemiptera: Auchenorrhyncha: Cicadomorpha). Zootaxa, 3895: 1-30.

Falaschi, R.L.; Oliveira, S.S. \& Lamas, C.J. 2016. Catalogue of Anisopodidae (Diptera, Bibionomorpha) types housed in the collection of the Museu de Zoologia da Universidade de São Paulo, Brazil. Revista Brasileira de Entomologia, 60(1): 24-29.

Falaschi, R.L.; Oliveira, S.S. \& Lamas, C.J.E. 2018. Catalogue of Bibionidae (Diptera: Bibionomorpha) types housed in the collection of the Museu de Zoologia da Universidade de São Paulo, Brazil. Papéis Avulsos de Zoologia, 58: 1-5.

Fernandes, J.A.M. \& Grazia, J. 2011. In: Fernandes, J.A.M.; Grazia, J. \& Campos, L.D. 2011. Redescription of Callostethus Ruckes, 1961 (Hemiptera: Heteroptera: Pentatomidae: Discocephalinae) with description of C. flavolineatus sp. nov. Zootaxa, 2866: 55-60.

Ferreira, V.S.; Prado, L.P. \& Seripierri, D. 2016. The entomological collection of Ricardo von Diringshofen (1900-1986) and its incorporation to the Museu de Zoologia da Universidade de São Paulo. Revista Brasileira de Entomologia, 60(2): 117-122.

Fortes, N.D.F. \& Grazia, J. 2000. Novas espécies do gênero Rio (Heteroptera, Pentatomidae). Iheringia, Série Zoologia, (88): 67-102.

Fortes, N.D.F. \& Grazia, J. 2005. Revisão e análise cladística de Serdia Stål (Heteroptera, Pentatomidae, Pentatomini). Revista Brasileira de Entomologia, 49: 294-339.

Gil-Santana, H.R. 2015. First record of the genus Pseudopothea from South America, with description of a new species from Brazil (Hemiptera: Heteroptera: Reduviidae: Ectrichodiinae). Zootaxa, 3904(4): 541-552.

Gil-Santana, H.R \& Baena, M. 2009. Two new species of Brontostoma Kirkaldy (Hemiptera: Heteroptera: Reduviidae: Ectrichodiinae) from Bolivia, with description of the male genitalia of two other species of the genus, and description of the female of $B$. doughertyae Gil-Santana, Lopes, Marques \& Jurberg. Zootaxa, 1979: 41-52. 
Gil-Santana, H.R. \& Forero, D. 2009. A new species of Notocyrtus, a new synonym of Coilopus, and new records and notes on other Harpactorini (Hemiptera: Heteroptera: Reduviidae: Harpactorinae) from South America. Zootaxa, 2148: 55-67.

Grazia, J. 1967. Estudos sobre 0 gênero Galedanta Amyot \& Serville, 1843 (Hemiptera-Heteroptera, Pentatomidae). Iheringia, Série Zoologia, (35): 45-49.

Grazia, J. 1978. Revisão do gênero Dichelops Spinola, 1837 (Heteroptera, Pentatomidae, Pentatomini). Iheringia, Série Zoologia, (53): 3-119.

Grazia, J. 1980. Revisão do gênero Pallantia Stål, 1862 (Heteroptera, Pentatomidae). Revista Brasileira de Entomologia, 24(1): 15-27.

Grazia, J. 1983. Sobre 0 gênero Phalaecus Stål, 1862 com a descrição de quatro novas espécies (Heteroptera, Pentatomidae). Revista Brasileira de Entomologia, 27(2): 177-187.

Grazia, J. 1987. Duas novas espécies de Euschistus do subgênero Mitripus Rolston, 1978 (Heteroptera, Pentatomidae, Pentatomini). Revista Brasileira de Entomologia, 31(1): 83-88.

Grazia, J. \& Barcellos A. 1994. Neotibilis, um novo gênero de Pentatomini (Heteroptera). Iheringia, Série Zoologia, (76): 55-94.

Grazia, J. \& Campos, L.A. 1996. Hypanthracos, um novo gênero de Pentatomini (Heteroptera: Pentatomidae). Iheringia, Série Zoologia, (80): 13-19.

Hildebrand, R. \& Grazia, J. 1983. Uma nova espécie de Euschistus Dallas, 1851 (Heteroptera, Pentatomidae). Iheringia, Série Zoologia, (62): 81-88.

Knight, H.H. 1917. A revision of the genus Lygus as it occurs in America North of Mexico, with biological data on the species from New York. Bulletin Cornell University Agricultural Experiment Station, 391: 557-645.

Kormilev, N.A. 1968. Notes on Neotropical Aradidae XVIII (HemipteraHeteroptera). Papéis Avulsos de Zoologia, 22: 47-56.

Kormilev, N.A. 1976. A New Genus and Seven New Species of Neotropical Aradidae (Hemiptera: Heteroptera). Revista Brasileira de Biologia, 36: 735-743.

Landim, M.I. 2011. Museu de Zoologia da Universidade de São Paulo: adaptação aos novos tempos. Estudos Avançados, 25(73): 205-216.

Lent, H. 1955. Revisão dos "Piratinae" Americanos. I. 0 genero "Tydides" Stal, 1865 (Hemiptera, Reduviidae). Revista Brasileira de Biologia, 15: 159-176.

Longo, R.S.; Ribeiro, J.R.I. \& Nessimian, J.L. 2005. A new species of Coleopterocoris Hungerford from southeastern Brazil, with notes on C. hungerfordi De Carlo and C. kleerekoperi Hungerford (Hemiptera: Heteroptera: Potamocoridae). Zootaxa, 1016: 39-47.

Moreira, F.F.F. \& Barbosa, J.F. 2012. Two new species of Paravelia Breddin, 1898 and distributional notes concerning the Veliidae from Minas Gerais State, Brazil (Insecta: Hemiptera: Heteroptera: Gerromorpha). Zootaxa, 3354: 58-68.

Moreira, F.F.F.; Nessimian, J.L.; Rúdio, J.A. \& Salles, F.F. 2010. New species and new records of Veliidae from Espírito Santo State and adjacent Minas Gerais State, Brazil, with notes on nomenclature (Insecta: Heteroptera: Gerromorpha). Journal of Natural History, 44: 2761-2801.

Nemésio, A. \& Melo, A.L. 2012. Nomenclatural issues in Rhagovelia Mayr (Hemiptera: Heteroptera: Gerromorpha: Veliidae). Zootaxa, 3230: 65-66.

Nieser, N. 1994. A new species and a new status in Neogerris Matsumura (Heteroptera: Gerridae) with a key to American species. Storkia, 3: 27-37.

Nieser, N. \& Melo, A.L. 1997. Os Heterópteros Aquáticos de Minas Gerais - Guia Introdutório com Chave de Identificação para as Espécies de Nepomorpha e Gerromorpha. Belo Horizonte, Editora UFMG. 180p.

Nieser, N. \& Pelli, A. 1994. Two new Buenoa (Heteroptera: Notonectidae) from Minas Gerais (Brazil). Storkia, 3: 1-4.

Onody, H.C.;Kawada, R.\& Brandão, C.R.F. 2014. Type specimens of Hymenoptera deposited In the Museu de Zoologia da Universidade de São Paulo, Brazil (excluding the Aculeata). Papéis Avulsos de Zoologia, 54: 391-402.

Packauskas, R.J. \& Schaefer, C.W. 1998. Revision of the Cyrtocoridae (Hemiptera: Pentatomoidea). Annals of the Entomological Society of America, 91: 363-386.
Polhemus, D.A.\& Carrie, R.H. 2013. A new species of Potamocoris (Heteroptera: Potamocoridae) from Belize, and synonymy of the genus Coleopterocoris. Tijdschrift voor Entomologie, 156(2-3): 141-149.

Prosen, A.F. \& Martinez, A. 1953. Una nueva espécie de Agriocleptes (Hemiptera: Reduviidae: Apiomerinae). Misión de Estudios de Patología Regional Argentina, 24: 53-56.

Prosen, A.F. \& Martínez, A. 1955. Una nueva especie de Apiomerus (Hemiptera). Misión de Estudios de Patología Regional Argentina, 26: 43-46.

Rodrigues, H.D.D.\& Moreira, F.F.F. 2016a. A new species and new records of Oiovelia (Heteroptera: Gerromorpha:Veliidae) from Brazil. Zootaxa, 4078(1): 153-160.

Rodrigues, H.D.D. \& Moreira, F.F.F. 2016b. A new species, new synonymy, and notes on Paravelia Breddin (Hemiptera: Heteroptera: Veliidae). Papéis Avulsos de Zoologia, 56(17): 183-188.

Rodrigues, H.D.D. \& Moreira, F.F.F. 2016c. Four new species of Paravelia (Hemiptera: Heteroptera: Veliidae) from Brazil, with notes on previously described species. Canadian Entomologist, 148: 642-667.

Rodrigues, H.D.D.; Melo, A.L. \& Ferreira-Keppler, R.L. 2014a. Taxonomic revision of the Neotropical genus Oiovelia (Hemiptera: Heteroptera: Veliidae). Acta Entomologica Musei Nationalis Pragae, 54(1): 65-98.

Rodrigues, H.D.D.; Moreira, F.F.F.; Nieser, N.; Chen, P.P.; Melo, A.L.; Dias-Silva, K. \& Giehl, N.F.S. 2014b. The genus Paravelia Breddin, 1898 (Hemiptera: Heteroptera: Veliidae) in Brazil, with descriptions of eight new species. Zootaxa, 3784: 1-47.

Rodrigues, H.D.D.; Sites, R.W. \& Giehl, N.F.S. 2016. Revision of the Neotropical genus Carvalhoiella De Carlo (Heteroptera: Nepomorpha: Naucoridae). Zootaxa, 4138(3): 577-590.

Rolston, L.H. 1983. The genus Paralincus (Hemiptera: Pentatomidae). Journal of the New York Entomological Society, 91: 183-187.

Santos, A.D.D.;Onody,H.C.\&Brandão,C.R.F.2017. Typespecimens ofChrysidoidea (Hymenoptera) deposited in the Museu de Zoologia da Universidade de São Paulo, Brazil. Papéis Avulsos de Zoologia, 57(26): 327-345.

Schuh, R.W. \& Slater, J.A. 1995. True bugs of the world (Hemiptera: Heteroptera): Classification and natural history. Ithaca, Cornell University Press. 336p.

Silva, E.J.E.; Fernandes, J.A.M. \& Grazia, J. 2006. Caracterização do grupo Edessa rufomarginata e descrição de sete novas espécies (Heteroptera: Pentatomidae: Edessinae). Iheringia, Série Zoologia, 93: 345-362.

Spangler, P.J. 1986. Two new species of water-striders of the genus Oiovelia from the tepui Cerro de la Neblina, Venezuela (Hemiptera: Veliidae). Proceedings of the Entomological Society of Washington, 88: 438-450.

Spangler, P.J. 1989. A new species of neotropical water bug, Paravelia biae, from Brazil (Heteroptera: Veliidae). Proceedings of the Entomological Society of Washington, 91: 360-366.

Taddei, V.A.; Martins, U.R.; Vivo, M. \& Percequillo, A.R. 1999. Museus e Coleções Zoológicas. In: Brito, M.C.W. \& Joly, C.A. (Orgs.). Biodiversidade do Estado de São Paulo. São Paulo, Winner Graph. v. 7, p. 49-67.

Ulysséa, M.A.;Prado, L.P.\& Brandão, C.R.F. 2015. Type specimens of the traditional Myrmicinae (Hymenoptera: Formicidae) ant tribes deposited in the Museu de Zoologia da Universidade de São Paulo, Brazil: Adelomyrmecini, Basicerotini, Blepharidattini, Crematogastrini, Formicoxenini, Lenomyrmecini, Myrmicini, Phalacromyrmecini, Pheidolini, Stegomyrmecini, Stenammini and Tetramoriini. Papéis Avulsos de Zoologia, 55(12): 175-204.

Valim, M.P. 2009. Type specimens of lice (Insecta: Phthiraptera) held in the Museu de Zoologia da Universidade de São Paulo, Brazil. Papéis Avulsos de Zoologia, 49(17): 197-219.

Weirauch, C. \& Schuh, R.T. 2011. Systematics and evolution of Heteroptera: 25 years of progress. Annual review of Entomology, 56: 487-510.

Wygodzinsky, P. 1945. Notas e descrições de "Emesinae" neotropicais (Reduviidae, Hemiptera). Revista Brasileira de Biologia, 5(2): 247-262.

Wygodzinsky, P. \& Schmidt, K. 1991. Revision of the new world Enicocephalomorpha (Heteroptera). Bulletin of the American Museum of Natural History, 200: 1-265. 